\title{
Idiographische und nomothetische Forschung in wissenschaftstheoretischer Sicht
}

\author{
C. Hubig*
}

Gemä $\beta$ der traditionellen Haltung, da $\beta$ nicht das Etablierte, sondern Vorschläge zur Einführung von Neuerungen unter Legitimationszwang stehen, sieht sich jede neue Forschungsrichtung dem Raster der alten Klassifikationen gegenübergestellt und inquisitorisch nach der Selbstverortung und der damit zusammenhängenden Ubernahme eines Legitimationsangebots befragt. Abgesehen von der Fragwürdigkeit dieses Verfahrens, das sich nur zirkulär selbst zu begründen vermag, ist die biographische Methode, die im hiesigen Zusammenhang diskutiert werden soll, bereits ein erfahrener Kandidat dieser Prüfung, der sich vorschnellen Charakterisierungen und Reduktionismen schon öfter auf raffinierte Weise entzogen hat. Dies allerdings oft dadurch, da $B$ sie seit ihrer programmatischen Begründung durch Petrarca dazu tendierte, sich in Bereiche außerhalb der etablierten Disziplinen zu begeben, etwa den literarischen oder allenfalls denjenigen literarischer Hermeneutik, die trotz allen programmatischen Getöses in den realen Geisteswissenschaften weniger verankert ist, als man annehmen mag, da man sich dieser strengen Kunst allzuleicht durch reduzierte Methodenideale in der Art von Kochrezepten zu entziehen vermag. Petrarca (vgl. dazu Abschn. 3) hat die Biographik als individualisierendes Prinzip einer Biographik gegenübergestellt, die im Dienste von Repräsentation und Legitimation ihre Gegenstände schematisch auf Paradigmen bezog, auf Archetypen historischen Heldentums und historischer Bedeutsamkeit. Jene normative Allgemeinheit war konfrontiert mit dem selektiven Vorgehen der Annalengeschichtsschreibung, deren Sammelsurium von Einzelheiten den gleichen Zweck auf der Ebene historischer Einzeltatsachen zu erfüllen hatte. Die Aufwertung des historischen Sinnes (a) durch die neuzeitliche Geschichtswissenschaft - im Mittelalter kam ihm nur Bedeutung zu als Ausgangspunkt allegorischer (b), tropologisch-ethischer (c) und anagogisch-mystischer (d) Deutung („vierfacher Wortsinn“) - zog nun die Biographik in das neue Spannungsfeld zwischen der Inanspruchnahme von Biographien als Gegenstand empirischer Geschichtswissenschaft und ihrer Bedeutung als Instanz der Selbstvergewisserung in den interpretierenden Wissenschaften. Je nach ihrem Selbstverständnis wiederholte sich innerhalb der Psychologie derselbe Konflikt, zusätzlich bereichert durch die Auffassungen von Psychologie als Gesetzeswissenschaft, gegenüber der die Biographik dann einen bloßen Anwendungsfall ausmacht.

- Möckernstraße 78, 1000 Berlin 61. 


\section{Idiographik und Nomothetik}

Wilhelm Windelband orientierte seinen Versuch einer Klassifikation der Wissenschaften, der in einer langen Reihe ähnlicher Versuche um die Jahrhundertwende steht, an ihren Erkenntniszielen. Wenn eine Wissenschaft „das Allgemeine in der Form eines Naturgesetzes" zu erfassen sucht, sollte sie "nomothetisch“" genannt werden, wenn sie hingegen das „Einzelne in der geschichtlich bestimmten Gestalt" suche, „idiographisch“ (Windelband 1911, S.145). Diese Gegenüberstellung von „Gesetzeswissenschaften“ und „Ereigniswissenschaften“ dient ihm dazu, den Geschichtswissenschaften gegenüber den Naturwissenschaften eine eigenständige Position zuzuweisen. Damit wendet er sich auch gegen eine Aufteilung der Erfahrungswissenschaften in Natur- und Geisteswissenschaften, da diese übersehe, daß die zentrale Geisteswissenschaft, die Psychologie, eine nomothetische sei. Nicht nach ihrem Gegenstand, sondern lediglich nach der Form ihrer Begrifflichkeit seien daher die Wissenschaften zu sondern. Daher erkennt Windelband auch keine Verschiedenheit in den Methoden an: Die kausale Erklärung sei für beide Bereiche die maßgebliche, und es bedürfe nicht eines eigenen Verstehenskonzeptes für die Geschichtswissenschaften, denn die ,idiographischen Wissenschaften [bedürfen] auf Schritt und Tritt der allgemeinen Sätze, die sie in völlig korrekter Begründung nur den nomothetischen Wissenschaften entlehnen können" (S.156). Sucht man also die spezifischen Unterschiede auf der Ebene der Begrifflichkeit, so führt dies in den methodischen Monismus kausalen Erklärens, da dieses gerade Einzelheit und allgemeines Gesetz aufeinander bezieht. Allerdings dürfe man, so Windelband, aus den Geschichts- keine Naturwissenschaften machen, da man sonst nur „triviale Allgemeinheiten“ erhielte (S.155). Damit versucht Windelband der Tatsache gerecht zu werden, da $B$,,alle lebendige Wertbeurteilung des Menschen an der Einzigartigkeit des Objekts hänge“, ohne aber nach deren wissenschaftlicher Verankerungsmöglichkeit zu fragen. Auch konstatiert er die „Merkwürdigkeit“, daß die Ansprüche der Geschichtswissenschaft an die Psychologie bisher zu „nachsichtig“ gewesen seien, andererseits der „unvollkommene Grad“, in dem bisher die „Gesetze des Seelenlebens“ hätten formuliert werden können, die Historiker nicht gehindert habe, durch Intuition und Menschenkenntnis ihre Helden und Handlungen adäquat zu verstehen (S.157). Was ist dies aber dann für eine „Menschenkenntnis"?

So sehr sich die von Windelband geprägte Unterscheidung im Methodendiskurs eingebürgert hat, so sehr offenbaren sich bereits bei ihrem Begründer ihre Schwächen. Daran ändert auch die Tatsache nichts, daß man heutzutage in raffinierterer Terminologie denselben Fehler begeht. Die Leistungsfāhigkeit methodischer Vorgaben ist daran zu bemessen, inwieweit sie anerkannte wissenschaftliche Erträge (qua Rekonstruktion) zu begründen und dadurch methodische Regeln zu entwickeln erlauben, unter denen vergleichbare Resultate gewonnen werden können. Ưber die Kriterien der Anerkennung hat nicht die Methode selbst zu entscheiden. 


\section{Individuelle Gesetze?}

Die biographische Methode findet in der eben eröffneten methodischen Alternative nicht ihren Ort. Denn die Begriffe, mit denen eine Biographie erstellt wird, beziehen sich weder auf bloB Individuelles als Anwendungsfall von Gesetzen im Sinne einer Typisierung des Individuellen oder einer Exemplifikation des Allgemeinen noch suchen sie eine Spezifik unter Absehung allgemeiner Eigenschaften. Nur solche nämlich machen eine Biographie überhaupt zugänglich. Dasselbe gilt für die Begrifflichkeit der Biographik selbst, deren Gegenstand die individuellen Biographien sind, die durch eine Allgemeinheit ihrer Begrifflichkeit erst zu einem diskutierbaren Gegenstand werden. Selbst wenn die Biographik die Biographien schematisiert und in Theorien verankert, so ist sie sich der Tatsache bewußt, daß ihr Gegenstand als Resultat einer Handlung der Beschreibung, die sich auf Handlungen der Realisierung von Zwecken des Lebens bezieht, einer doppelten intentionalen Analyse bedarf - der Rekonstruktion der Handlungsprämissen des individuellen Lebens und der Prämissen seiner Beschreibung. Bevor diese Frage weiter verfolgt werden soll, ist jedoch aus der Sicht der moderneren Wissenschaftstheorie der Versuch zu diskutieren, durch ein ausdifferenzierteres Verfahren die Idee kausalen Erklärens für den Bereich der Historie, somit auch der biographischen Verfahren gegenüber dem Damoklesschwert eines scheinbaren Irrationalismus der Hermeneutik zu bewahren. Die Versuche bestehen zum einen darin, den Anspruch deduktiv-nomologischen Erklärens überhaupt zu erhalten, oder ihn soweit abzuschwächen, daß der Erklärungsanspruch ersetzt wird durch einen Beschreibungsanspruch, der dann aber - im Sinne einer Reduktion der wissenschaftlichen Interessen - für das ganze gelten soll. Zum andern wird das erklärende Gesetz abgelöst durch die Behauptung des Vorliegens einer Regel, die unter bestimmten Bedingungen Gesetzescharakter annehmen kann.

Gegenüber dem Versuch, historische Einzelheiten, deren Beschreibung eine Biographie ausmacht, deduktiv-nomologisch zu erklären, haben William Dray, Gilbert Ryle und M. Scriven die wichtigsten Argumente vorgetragen. Bekanntlich besteht eine deduktiv-nomologische Erklärung darin, $\mathrm{da} B$ unter der gegebenen Prämisse eines allgemeinen Gesetzes sowie einer Prämisse, die das Vorliegen der Antezedensbedingungen formuliert, die die Wenn-Komponente des Gesetzes realisieren, das zu erklärende Ereignis als Realisierungsfall der Dann-Komponente des Gesetzes logisch abgeleitet wird (Hempel 1977). Im Falle historischer Explananda steigt die Anzahl der Antezedensbedingungen in sehr hohem Maße, was dazu zwingt, die Wenn-Komponente des Gesetzes entsprechend zu modifizieren, so $\mathrm{da} B$ sie ungeheuer komplex wird, und das Gesetz letztendlich nur noch einen einzigen Anwendungsfall - eben das zu erklärende Ereignis - hat (Dray 1957, 1975). In diesem Fall kōnne man aber nicht mehr von Gesetzen sprechen. Hier läge also ein Plädoyer für eine ideographische Ausrichtung der Biographik in anderer Form, als sie Windelband noch vorsah, vor. Anstelle eines Rekurses auf Gesetzesprämissen, den Windelband immer noch forderte, verweist Dray auf sogenannte SchluBregeln des Historikers, die ihm erlauben, Erklärungen der Form

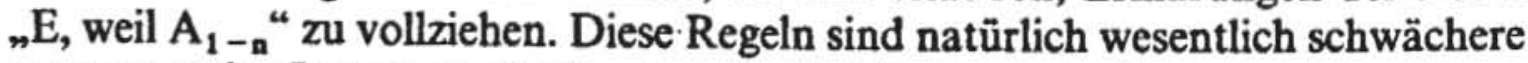
argumentative Instanzen als Gesetze, die Ableitungen erlauben. 
Ryle (1950) argumentiert in dieselbe Richtung, wenn er auf den grundlegenden Unterschied zwischen Aussagen der Art "wenn p, dann q" und "q, weil p“ hinweist. Sätze der ersteren Form bezeichnet er als "general hypotheticals", die aufgrund ihrer konditionalen Form in ihren Wahrheitsbedingungen nicht durch Gegenstandsbezug, sondern nur dadurch bestimmt werden können, daß die Überzeugung von ihrer Wahrheit bestimmte Argumentationsweisen festlegt. Dagegen wenden die Verteidiger deduktiv-nomologischer Erklärung ein (Stegmüller 1983, S. 140), daß selbst in dem Falle, wo dem Gesetz nur eine einzige Anwendung entspräche, zumindest theoretisch weitere Anwendungsfalle denkbar wären, was sich insbesondere auf die Prognoseleistung der deduktiv-nomologischen Erklärung auswirke. Allerdings müsse darauf geachtet werden, daß die komplexe Gesetzesprāmisse nicht logisch äquivalent mit dem Explanandum wird.

Abgesehen davon, daß sich dies nur in den wenigsten Fällen vermeiden ließe, gibt es dagegen einen grundsätzlichen Einwand: Die deduktiv-nomologische Struktur von Erklärungen unter Hinweis auf ihre potentielle Prognoseleistung zu stützen, impliziert die Grundannahme einer logisch-einheitlichen Struktur von Erklärung und Prognose. Prognosen sind aber auch möglich, wenn hinreichende Bedingungen angenommen werden, ohne da $B$ ein notwendiger Bedingungszusammenhang geschweige denn eine (Natur-) Gesetzlichkeit mit Erklärungskraft angenommen wird. So kann ein erfolgreiches Prognostizieren auf Erfahrungsregeln beruhen, die, ohne daß man sich dessen bewußt wäre, die bekannten "selffulfilling"- bzw. "suicidal"-Eigenschaften von "prophecys" bereits mit berücksichtigen (Merton 1971). Von daher erscheint es unbegründet, auf insgeheim unterstellte Gesetzesprämissen zu schließen, wenn eine Prognose erfüllt wurde, und eine latente deduktiv-nomologische Erklärungsstruktur zu unterstellen.

Scrivens (1975) Kritik an diesen Bemühungen geht noch weiter, indem er für den Fall der Historie „p, weil q“-Aussagen nicht einmal mehr als Argumente, sondern als bloße Sätze rekonstruiert. In solchen erklärenden Aussagen würden nur Einzeltatsachen erwähnt und erst auf die Frage nach einer Begründung für diese „Erklärung“ würden relevante Gesetzmäßigkeiten nicht als zusätzliche Prämissen, sondern als "role justifying grounds" angeführt. Gesetze sind also nicht unmittelbar erklärungsrelevant, sondern ihre Annahme rechtfertigt eine kausale Erklärung. Wichtig ist, zu berücksichtigen, daß eine Rekonstruktion nicht den erklärenden singulären Weil-Satz als singuläre Wenn-dann-Aussage interpretieren darf. Denn dann läge in der Tat eine bloß unvollständig rekonstruierte Fassung einer deduktiv-nomologischen Erklärung vor.

Auch eine Modifikation des deduktiv-nomologischen Modells in sogenannte genetische Erklärungen kann die Ansprüche einer Biographik nicht erfüllen. Das Spezifikationserfordernis gegenüber den Antezedensbedingungen wird hier zwar durch eine zeitliche Indizierung entsprechend der Indizierung des Explanandum gegeben und durch die Verkettungsmöglichkeit verschiedener deduktiv-nomologischer Modelle werden kausale Ableitungen relativiert, jedoch bleibt die grundlegende Problematik der Annahme von Gesetzen als Prämissen erhalten. Das Problem verschärft sich noch, wenn man sich in Erinnerung ruft, daß viele sogenannte "Gesetze“ im historischen Bereich in ihrer Wenn-Komponente Eigenschaften anführen, die Dispositionen darstellen. Dispositionen sind Eigenschaften, die die Realisierung eines Zustandes unter bestimmten Bedingungen darstel- 
len, was durch sogenannte Reduktionssätze rekonstruierbar ist. Die Wahrheit der Dispositionszuschreibung ist äquivalent mit der Wahrheit des Reduktionssatzes, der als empirischer Satz den Zusammenhang zwischen Realisierungsbedingungen und eintretendem Effekt beschreibt. Im Gegensatz zu naturwissenschaftlichen Dispositionsprädikaten läßt sich jedoch für den Bereich menschlicher Handlungen zeigen, da $B$ die Rekonstruktion von Dispositionsprädikaten, als die bestimmte mentalistische Kategorien des Wollens, Wünschens und Erwartens rekonstruiert werden können, zu analytischen Sätzen führen, so daß die Gesetzesprämisse ihres empirischen Charakters entkleidet und zu einer bloßen Definition wird. Diese ist abhängig von sprachlichen Konventionen als Ausdruck sozialer Orientierungssysteme (Hubig 1985 a, S. 66 ff.).

Eine letzte Möglichkeit, jene paradoxen individuellen Gesetze, von denen die Möglichkeit eingeschränkt deduktiv-nomologischer Erklärungen abhängt, zu erhalten, besteht darin, sie als Setzungen des Individuums, dessen Handeln unter ihnen erklärt werden soll, zu verstehen. Georg Simmel (1913) versuchte auf der Basis solcher Gesetze als individueller Handlungsideale insbesondere das Zustandekommen von Kunstwerken zu erklären. Allerdings erscheint bei näherer Betrachtung der Begriff des Gesetzes hier als unangemessen, da sie lediglich eine $z u$ sätzliche Antezedensbedingung als singuläres Ereignis meinen: die Tatsache nämlich, daß ein bestimmtes Individuum eine bestimmte Regel als nur für sich maßgeblich erachtet. Allerdings muß dieses Individuum sich selbst fiktiv verallgemeinern, weil es sonst seinen Regelvollzug nicht kontrollieren kann, d.h. keine Bezugsinstanz mehr hätte, die es als Handelnden in eine Relation setzt zu der Regel (Wittgenstein 1971, S. 258), denn der Zusammenhang zwischen Regel und dem durch sie Begründeten ist nicht der einer gegebenen Determination, sondern Resultat einer Interpretation. Eine solche Interpretation ist also Voraussetzung dafür, daß im nachhinein eine singuläre „Erklärung“ im Sinne einer Aussage (Scriven) getätigt werden kann. Der Gegensatz nomothetisch-idiographisch wird also abgelöst - wenn das Wortspiel erlaubt sein soll - durch einen Begründungszusammenhang normothetisch-idiographisch.

\section{Petrarcas programmatische Begründung der Biographik}

In seinen „Freundesbriefen“, die als früheste Zeugnisse der Herausbildung eines neuzeitlichen Konzepts von Individualität gelten, schildert Petrarca (1336-1350) die Umstände, die ihn veranlassen, eben diese alten Briefe zu versenden, die größtenteils aus Notizen und Entwürfen in Kontext mit Erlebnissen seines bisherigen Lebens stehen. An Sokrates (S. 220) beschreibt er sich als einen Wanderer, der von der Warte seines Zieles aus rückwärts schaut, anstatt, wie er es ursprünglich vorhatte, die unvollkommenen Entwürfe der davor liegenden Zeit zu vernichten. Unter dem mittelalterlichen Ideal der perfectio wäre die Akzidentialität dieser Entwürfe evident und ihnen keinerlei Wert zuzusprechen. 300 Jahre vor der berühmten "Querelles des anciens et des modernes" benutzt Petrarca nun aber diese Spuren (S.230), um sich über Höhen und Tiefen seines Lebens zu vergewissern und somit die Idee einer perfectabilité, also Vervollkommnungsfähigkeit im ästhetischen, historisch-technischen und sozialen (kommunikativen) Sinne zu exemplifi- 
zieren. Genau dies war das Thema jener "Querelle", auf deren umstrittener Kategorie der perfectabilité sich das neue Kunst- und Geschichtsverständnis gründen sollte (Jauss 1964). Die Flüchtigkeit des Lebens angesichts des Todes aufzuhalten (S.221), um Versprechungen an andere Subjekte und ihre Hoffnungen einzulösen, veranlassen Petrarca, explizit Unvollkommenes, nicht Abgeschlossenes, Unbewältigtes zum Gegenstand seiner Schilderung in einem zusammenfassenden Konvolut zu machen. Als zweiter Odysseus sieht er sich (S. 225), der Glanz beider Namen und ihre Identität gründe sich einzig auf den Erfahrungen ihrer unsteten Wanderschaft, nicht einer vorgegebenen Rolle in einem Orientierungssystem, das eine biographische Selbstvergewisserung erübrigt. Odysseus ist bekanntlich das Paradigma eines Wesens, das, seiner vorgegebenen Identität verlustig, diese erst durch Erzählung wiedergewinnen kann - das Paradigma rationaler Individualität überhaupt.

$\mathrm{Da} B$ alles Biographische nun an einer Stelle zusammenkommt, ließe am Gesamtkörper erkennbar werden, was in den einzelnen Gliedern verdeckt war (S. 225). Wiederholungen würden deutlich sowie stilistische Inhomogenitäten als durch die Notwendigkeit bedingt erkennbar, $\mathrm{da} B$ die verschiedensten geistigen Ansprüche in den Wechselfällen seines Lebens verschiedene Darstellungsformen erforderten. An der Art dieser Darstellungen könne man seinen jeweiligen Geisteszustand erkennen. Leicht könne man sich darüber lustig machen, wie jemand, der untätig am Ufer sitzt, über die Steuermannskunst urteile. Jene Allegorie verdeutlicht den Handlungscharakter biographischer Bemühungen: „Habe ich aber erst einmal die letzte Hand an jenes andere Werk gelegt, so sollst Du es ... als das mit vielem Eifer ausgehauene Ebenbild meines Geistes... aufstellen“ (S. 230).

Hier soll gerade nicht unterstellt werden, daß etwas Vergleichbares wie Phidias' Minerva geschaffen werden sollte. Das Ende seiner biographischen Bemühungen werde mit dem Ende seines Lebens zusammenfallen. Im Kontext der Schilderung seiner berühmten Wanderung auf den Mont Ventoux (1336), einem Erlebnis, dessen Eindruck einer überfordernden Erhabenheit der Natur ihn auf sich selbst zurückwarf, schildert er rückblickend in raffinierter Stilisierung nochmals die Motivation zum Biographischen. Die Unermeßlichkeit des Raumgefühls habe sich auf sein Zeitgefühl übertragen, der Eindruck des unbewältigten Wandels, deutlich noch als Widerstreit der Menschen in ihm, habe ihn gezwungen, aus dieser zeitentrückten Perspektive des Gipfelerlebnisses sich auf sich selbst zu wenden. Zufällig habe sich ihm beim Aufschlagen seiner Reiselektüre, der „Bekenntnisse“ des Augustinus, jene Stelle präsentiert: „Und es gehen die Menschen, zu bestaunen die Gipfel der Berge und die ungeheuren Fluten des Meeres und die weit dahinfließenden Ströme und den Saum des Ozeans und die Kreisbahnen der Gestirne, und haben nicht acht ihrer selbst“ (1336, S.259). Diese Stelle aber, von ihm wohl vorher viel gelesen, wird nun in parabolischer Weise gegensinnig interpretiert. War es für Augustinus ein Appell, sich von der Äußerlichkeit der Welt auf das Göttliche im Menschen zu konzentrieren, so wird für Petrarca das erstmals reale Erlebnis unermeBlicher Natur an der Epochenschwelle zum AnlaB, sein inneres Auge auf sich selbst zu richten. Folgendes ist hervorzuheben: die Entgrenzungsgefahr veranlaBt das Subjekt in vergleichbarer Weise, wie sie Kant in der „Kritik der Urteilskraft" rekonstruiert hat, sich zu reflektieren, um sich seiner eigenen (beschränkten) Freiheit zu versichern. Damit ist das praktische Interesse 
der Biographik vorgegeben. Diesem Interesse steht die Wandelbarkeit der Zeit und der Widerstreit der Identitäten in uns entgegen. Erst eine sorgsame Rekonstruktion der Entwicklung kann aus diesem Dilemma führen. Gegenstand dieser Rekonstruktion ist jedoch nicht das reale Leben, sondern seine Spuren, die in (meist schriftlichen) Zeichen aufbewahrt sind. Biographie ist also Zeichen von Zeichen. Letzteren kommt nur Bedeutung zu als Elementen und Trägern eines Gesamtzusammenhanges unter dem genannten praktischen Interesse. Die Idee von Biographik leitet sich also ab aus der Idee von Freiheit, die ihrerseits der Biographie bedarf, um sich ex negativo auf dem Wege über die Unvollkommenheiten des Lebens spurenhaft zu finden. Daher mündet dieses Konzept in eine neuzeitliche Selbstbescheidung der "devotio“ bzw. „pietas moderna“, die der „idiota“der Laie - des Nikolas von Kues mit ähnlichem Argument erreicht. Das Ideal der Freiheit kann nur die Resignation umgehen, wenn es die Realität des Lebens im Sinne dieser Selbstbescheidung sich als Proze $B$ und nicht als entratenes Resultat vergegenwärtigt.

\section{Folgerungen}

Heinrich Rickert trug gegen Windelbands Klassifikation vor, daß die Spezifik historischen Denkens nicht an seiner idiographischen Ausrichtung, sondern in der Wertbeziehung als einer Erkenntnisintention liege, die das historisch Einzelne durch eben jene Relation bedeutsam mache (Rickert 1921). Max Weber (1904) vertiefte dieses Argument, indem er darauf hinwies, da $\mathrm{B}$ jegliche soziologische oder historische Erklärung von Handlungen aufgrund der intentionalen Struktur ihrer Prämissen durch einen Verstehensakt erst ermöglicht würde: Dieser beziehe das historisch Einzelne auf idealtypische „Wertideen“, die nicht als allgemeine Gattungsbegriffe gefaßt werden dürfen, somit auch keine Subsumption erlauben, sondern lediglich als ideale Grenzbegriffe, die quasi schematisch die kulturell verbindlichen Wertvorstellungen enthalten, unter denen die Zweckauswahl und der Mitteleinsatz der Handelnden stattfinden. Diese Idealtypen sind heuristische Hilfsmittel, deren Leistungsfähigkeit durch ihre Erklärungskraft in der historischen Konkretion erwiesen wird, die aber letztlich auf Wertvorentscheidungen des Forschers beruhen. Allerdings setzt dieses Modell, das die Verbindung von allgemeiner Betrachtungsweise und historischer Konkretion als Bedingungsverhältnis von Verstehen und Erklären faßt, grundsātzlich eine Rationalität des Handelns als Zweckrationalitāt voraus. Wenn diese nicht vorliegt, kapituliert der Erklärungsanspruch vor der Irrationalitāt der Handlung. Diese Einschränkung macht Max Webers Ansatz für die Entwicklung einer psychologischen Biographik problematisch.

Hier erscheint ein Rekurs auf Diltheys hermeneutischen Ansatz fruchtbarer. Dilthey (1907-1910, S. 191 ff.) weist nach, daß die Identifikation von Erlebnissen durch Ausgrenzung aus dem Erlebnisstrom nur durch Erlebnisausdrücke möglich ist, die dem historischen Subjekt verfügbar sind. Die Regeln der Zuordnung lassen sich - analog zu Kant - rekonstruieren als sog. materiale Kategorien, die die Rahmenvorstellung von Raum- und Zeitgefühl etc. für einen historischen Kontext oder historischen Typ ausmachen (dazu Hubig 1985a, b). Der Forscher 
selbst findet diese materialen Kategorien als Konstruktionsprinzipien in den Selbstbiographien beispielhaft vorgeführt (exemplifiziert). Jene biographisch orientierte Hermeneutik als „Aufstieg vom Allgemeinen zum Individuellen“ geht aus von den Selbstbiographien, rekonstruiert deren materiale Kategorien und vollzieht unter diesen die Zuordnung von Erlebnisausdrücken zu Erlebnissen nach. Dabei - und dies ist der praktische Zweck der Hermeneutik - erschließt sich der Hermeneutiker „Möglichkeiten“, die in seinem beschränkten eigenen Leben bisher nicht vorhanden waren. Jener Bereichungseffekt ist natürlich um so größer, wenn der Biographiker und der Biograph ein und diesselbe Person sind. Die Erinnerung an Petrarca sollte dies vor Augen führen. Er richtet sich unter den Ansprüchen der Biographik auf sein eigenes biographisches Material.

Die Biographik als Forschungsrichtung rekonstruiert also sowohl die Regeln, die in Biographien exemplifiziert sind, als auch die spezifische Art, wie diese Regeln im Leben des Biographierten modifiziert (dazu Hubig 1985a, S. $179 \mathrm{ff}$. sowie Kap. 3.3-3.4; 1985b, S. 338-348) werden. Allerdings wird diese Blickrichtung nicht durch einen direkten Bezug zum „Leben“ ermöglicht, denn dieses liegt ja nicht als Gegenstand, sondern nur in Zeichen vor. Auch dieser Blick ist also auf das Zeichenmaterial der Biographie angewiesen, sieht es jedoch unter dem Gesichtspunkt, der gerade nicht auf die Einstimmigkeit des Regelvollzug abhebt, sondern auf die Vielschichtigkeit und Widersprüchlichkeit der Äußerungen, in denen diese Regeln modifiziert werden.

Diese Dimension eröffnet sich nicht unter der Fragestellung, die das Besondere als Exemplifikation von Regeln, von „Weisen der Welterzeugung“ (Goodman) begreift. Sie ist nur zu erschließen durch die Analyse der Formen der Erlebnisausdrücke (z. B. die „schwache“ oder „starke“ Sprache bei Petrarca), also eine ästhetische Analyse im weitesten Sinne, die in diesen Formen die Spuren nicht des Daß der Intentionalität, sondern ihres Wie (des Schmerzes [Adorno], der „gehemmten Begierde“ [Hegel]), also dessen aufsucht, was sich als „Nichtidentisches“ dem eigentlich wissenschaftlichen Diskurs versperrt. Dennoch sind Verfahren denkbar, diese Dimension zu erschließen. Es sind begleitende Testverfahren, die die Authentizität eines Erlebnisausdruckes zu beurteilen erlauben. Auf solche Verfahren sowie die Notwendigkeit ihrer Verankerung in ethischen Prämissen - was im hiesigen Zusammenhang unter Hinweis auf Petrarca nur angedeutet werden konnte, habe ich an anderer Stelle hingewiesen (Hubig 1985b).

\section{Literatur}

Dilthey W (1907-1910) Gesammelte Schriften, Bd VII. Vandenhoek \& Ruprecht, Gōttingen 1958

Dray W (1957) Laws and explanation in history. Clarendon, London

Dray W (1975) Historische Erklärungen von Handlungen. In: Giesen B, Schmid M (Hrsg) Theorie, Handeln und Geschichte. Hoffmann \& Campe, Hamburg, S 261-283

Hempel G (1977) Aspekte wissenschaftlicher Erklärung. DeGruyter, Berlin New York

Hubig C (1978) Dialektik und Wissenschaftslogik. DeGruyter, Berlin New York

Hubig C (1985 a) Handlung, Identität, Verstehen. Beltz, Weinheim

Hubig C (1985b) Rationalitätskriterien inhaltlicher Analyse. In: Jüttemann G (Hrsg) Qualitative Forschung in der Psychologie. Beltz, Weinheim, S 327-350 
Jauss HR (1964) Ästhetische Normen und geschichtliche Reflexionen in der nQuerelle des Anciens et des Modernes". Fink, München

Merton R (1971) Die Eigendynamik gesellschafticher Voraussagen. In: Topitsch E (Hrsg) Logik der Sozialforschung. Kiepenheuer \& Witsch, Köln, S 144-163

Petrarca F (1336-1350) Dichtung und Prosa. Rūtten \& Loening, Berlin 1968

Rickert H (1921) Die Grenzen der naturwissenschaftlichen Begriffsbildung. Mohr, Tübingen

Ryle G (1950) "If", "So" and "Because". In: Black M (ed) Philosophical analysis. Prentice Hall, Ithaca, NY

Scriven M (1975) Truismen als Grundlage für historische Erklärungen. In: Giesen B, Schmid M (Hrsg) Theorie, Handeln und Geschichte. Hoffmann \& Campe, Hamburg, S 103-129

Simmel G (1904/1968) Das individuelle Gestz. Suhrkamp, Frankfurt

Stegmüller W (1983) Probleme und Resultate der Wissenschaftstheorie und analytischer Philosophie, Bd 1, 2. Aufl. Springer, Berlin Heidelberg New York

Weber M (1904) Methodologische Schriften. Fischer, Frankfurt 1968

Windelband W (1911) Präludien, Bd 2. Mohr, Tübingen

Wittgenstein L (1971) Philosophische Untersuchungen. Suhrkamp, Frankfurt 